\title{
Aliskiren ameliorates pressure overload-induced heart hypertrophy and fibrosis in mice
}

\author{
Li-qing WENG ${ }^{1, \#}$, Wen-bin ZHANG ${ }^{2, \#}$, Yong YE ${ }^{1, \#}$, Pei-pei $Y_{I N}^{1, \#}$, Jie YUAN ${ }^{1, \#}$, Xing-xu WANG ${ }^{1}$, Le KANG ${ }^{1}$, Sha-sha JIANG ${ }^{1}$, \\ Jie-yun YOU ${ }^{1}$, Jian $W^{1}$, Hui GONG ${ }^{1}$, Jun-bo GE ${ }^{1, *}$, Yun-zeng ZOU ${ }^{1, *}$ \\ ${ }^{1}$ Shanghai Institute of Cardiovascular Diseases, Zhongshan Hospital and Institute of Biomedical Science, Fudan University, Shanghai \\ 200032, China; ' Sir Run Run SHAW Hospital, Zhejiang University, Hangzhou 310016, China
}

\begin{abstract}
Aim: Aliskiren (ALK) is a renin inhibitor that has been used in the treatment of hypertension. The aim of this study was to determine whether ALK could ameliorate pressure overload-induced heart hypertrophy and fibrosis, and to elucidate the mechanisms of action. Methods: Transverse aortic constriction (TAC) was performed in mice to induce heart pressure overload. $\mathrm{ALK}\left(150 \mathrm{mg} \cdot \mathrm{kg}^{-1} \cdot \mathrm{d}^{-1}, \mathrm{po}\right)$, the autophagy inhibitor 3-methyladenine $\left(10 \mathrm{mg} \cdot \mathrm{kg}^{-1}\right.$ per week, ip) or the PKCßI inhibitor LY333531 $\left(1 \mathrm{mg} \cdot \mathrm{kg}^{-1} \cdot \mathrm{d}^{-1}, \mathrm{po}\right)$ was administered to the mice for 4 weeks. Heart hypertrophy, fibrosis and function were evaluated based on echocardiography, histological and biochemical measurements. Mechanically stretched-cardiomyocytes of rats were used for in vitro experiments. The levels of signaling proteins were measured using Western blotting, while the expression of the relevant genes was analyzed using real-time QRT-PCR. Results: TAC induced marked heart hypertrophy and fibrosis, accompanied by high levels of Ang II in plasma and heart, and by PKC $\beta I / \alpha$ and ERK1/2 phosphorylation in heart. Meanwhile, TAC induced autophagic responses in heart, i.e. increases in autophagic structures, expression of Atg5 and Atg16 L1 mRNAs and LC3-II and Beclin-1 proteins. These pathological alterations in TAC-mice were significantly ameliorated or blocked by ALK administration. In TAC-mice, 3-methyladenine administration also ameliorated heart hypertrophy, fibrosis and dysfunction, while LY333531 administration inhibited ERK phosphorylation and autophagy in heart. In mechanically stretched-cardiomyocytes, CGP53353 (a PKCßI inhibitor) prevented ERK phosphorylation and autophagic responses, while U0126 (an ERK inhibitor) blocked autophagic responses.

Conclusion: ALK ameliorates heart hypertrophy, fibrosis and dysfunction in the mouse model in setting of chronic pressure overload, via suppressing Ang II-PKCßI-ERK1/2-regulated autophagy.
\end{abstract}

Keywords: aliskiren; renin; cardiac hypertrophy; pressure overload; autophagy; angiotensin II; PKC; ERK1/2; 3-methyladenine; LY333531; cardiomyocyte

Acta Pharmacologica Sinica (2014) 35: 1005-1014; doi: 10.1038/aps.2014.45; published online 7 Jul 2014

\section{Introduction}

Cardiac hypertrophy can be prevented or regresssed in its initial stage ${ }^{[1,2]}$. If left neglected, however, it will ultimately progress into heart failure, a well-established risk factor significantly associated with increased cardiac morbidity and mortality $^{[3,4]}$. Cardiac hypertrophy is elicited by multiple factors, among which mechanical stress is an initial inducer ${ }^{[5]}$. It is therefore important to treat mechanical stress-induced cardiac hypertrophy. Hypertension is the disease most responsible for mechanical stress on the heart. Although antihypertensive

\footnotetext{
\# These authors contributed equally to this manuscript.

* To whom correspondence should be addressed.

E-mail jbge@zs-hospital.sh.cn (Jun-bo GE). zou.yunzeng@zs-hospital.sh.cn (Yun-zeng ZOU)

Received 2013-12-09 Accepted 2014-05-04
}

drugs, including angiotensin II (Ang II)-converting enzyme inhibitors (ACEI), AngII type 1 receptor (AT1-R) blockers $(\mathrm{ARB})$ and calcium channel antagonists, exert inhibitory effects on cardiac hypertrophy ${ }^{[6]}$, progression of the disease and subsequent cardiac dysfunction remain a significant problem in hypertensive subjects. As such, there is increasing interest in developing therapies offering more protection against hypertension-induced end-organ damage.

Aliskiren (ALK), a renin inhibitor, has a greater effect on hypertension than either an ACEI or an ARB because its most proximal locus of inhibition occurs at the rate-limiting step, avoiding a compensatory rise in downstream components of the renin-angiotensin-aldosterone system (RAAS) ${ }^{[7]}$. However, it is unclear whether ALK may effectively induce regression of cardiac hypertrophy. Although the combination of ALK with an ACEI or an ARB can synergistically attenuate both 
hypertrophy and dysfunction in hypertensive patient ${ }^{[8,9]}$, a systematic review of randomized, controlled clinical trials has shown that combined therapy is associated with a higher risk of hyperkalemia than monotherapy with an ACEI, an ARB or $\mathrm{ALK}^{[10]}$. In addition, some patients are intolerant of ACEI or $A R B$, whereas ALK is well tolerated ${ }^{[11]}$. Therefore, it is necessary to elucidate the effect of ALK monotherapy on pressure overload-induced cardiac hypertrophy, fibrosis and dysfunction, independent of $\mathrm{BP}$ reduction.

Cardiomyocyte autophagy was recently found to correlate with the development of cardiac hypertrophy ${ }^{[12,13]}$. Although the exact role of autophagy in the pathogenesis and progression from hypertrophied heart to heart failure is not completely understood, the increased autophagy reportedly parallels the extent of the resultant cardiac damage, which contributes to pressure overload-induced cardiac remodeling and subsequent heart failure.

Activation of protein kinase C (PKC) has been implicated in the pathogenesis of cardiac remodeling ${ }^{[14]}$. There are several subtypes of PKC. It has been determined that PKCa is both a predominant mediator of the hypertrophic response and a regulator of cardiac contraction ${ }^{[15]}$, whereas the role of PKC $\beta$ in cardiac hypertrophy and fibrosis in mice remains controversial $^{[16,17]}$.

The present study was carried out to observe whether ALK affects the development of cardiac hypertrophy and fibrosis in a mouse model in the setting of chronic pressure overload and to investigate whether myocardial autophagy is associated with the effects of ALK, as well as to determine the possible role of PKC in these effects.

\section{Materials and methods}

\section{Animal experiments}

Ten-week-old C57BL/6J male mice (The Jackson Laboratory, Bar Harbor, Maine, USA) were anesthetized before either transverse aorta constriction (TAC) or a sham operation was carried out as previously described ${ }^{[18]}$. In brief, the transverse aorta was constricted between the origins of the innominate artery and the left common carotid artery with a 7-0 nylon suture by ligating the aorta together with a blunted 27-gauge needle, which was removed later; a constriction of $0.4 \mathrm{~mm}$ in diameter was the product of this endeavor. ALK (150 $\mathrm{mg} \cdot \mathrm{kg}^{-1} \cdot \mathrm{d}^{-1}$, po, Novartis Pharma AG, Switzerland), autophagy inhibitor 3-methyladenine (3-MA, $10 \mathrm{mg}^{-\mathrm{kg}^{-1}}$ per week, ip, Santa Cruz Biotechnology, Santa Cruz, CA, USA) or PKC $\beta I$ inhibitor LY333531 (LY, 1 mg.kg-1 $\mathrm{d}^{-1}$, po, Axon Medchem BV, Groningen, Netherlands) was administered to mice for 4 weeks beginning on the day of operation. All animal experimental protocols were approved by the Animal Care and Use Committee of Fudan University and in compliance with Guidelines for the Care and Use of Laboratory Animals, published by the National Academy Press (NIH Publication No 85-23, revised 1996).

\section{Culture and mechanical stretch of cardiomyocytes}

The primary culture of cardiomyocytes was prepared as described previously ${ }^{[18]}$. The cells derived from the hearts of 1- to 2-d-old Sprague-Dawley rats were cultured in Dulbecco's modified Eagle's medium (DMEM) containing 10\% fetal bovine serum (FBS) and penicillin/streptomycin, and then incubated in serum and antibiotic-free conditions, in silicon-based plates pre-coated with collagen for $12 \mathrm{~h}$, after which time they were mechanically stretched by $20 \%$ for $24 \mathrm{~h}$ and subsequently collected for protein extraction. A PKCa inhibitor (Ro-31-0432, $200 \mathrm{pmol} / \mathrm{mL}$, Sigma-Aldrich, St Louis, USA), PKC $\beta$ I inhibitor (CGP53353, $6.0 \mathrm{nmol} / \mathrm{mL}$, Sigma-Aldrich, St Louis, USA) or ERK inhibitor (U0126, $10 \mu \mathrm{mol} / \mathrm{L}$, Cell Signal Technology, Danvers, MA, USA) was added to the culture medium $1 \mathrm{~h}$ prior to mechanical stretching.

\section{Echocardiography}

Four weeks after TAC, transthoracic echocardiography was performed on conscious, gently restrained mice as previously described using a $30 \mathrm{MHz}$ high frequency scan head (VisualSonics ${ }^{\circledR}$ Vevo770 ${ }^{\circledR}$, VisualSonics Inc., Toronto, Canada $)^{[19]}$. All measurements were averaged for five consecutive cardiac cycles.

\section{Morphological and histological analysis}

All mice were euthanized via neck dislocation while under anesthesia, and their hearts were surgically removed to calculate the heart to body weight ratio (HW/BW mg/g). For histological analysis, the ventricles were excised and fixed in $10 \%$ formalin for $48 \mathrm{~h}$ before being dehydrated using ethanol gradient and subsequently embedded in paraffin, sectioned at $4 \mathrm{~mm}$ thickness and stained with hematoxylin and eosin (HE), as well as Masson's trichrome (Sigma-Aldrich, St Louis, USA). Taken from six animals per group, a minimum of three sections per heart with five fields per section were analyzed to determine both the cross-sectional area (CSA) and fibrotic area of cardiomyocytes. All measurements were performed with an automated image analysis system (Image-Pro Plus 5.0, Media Cybernetics, USA).

\section{Transmission electron microscopy}

Freshly isolated heart tissue was fixed in ice-cold fresh 2.5\% glutaraldehyde fixative for $2 \mathrm{~h}$, rinsed in phosphate buffer, and subsequently post-fixed in $1 \% \mathrm{OsO}_{4}$ solution for another $2 \mathrm{~h}$. The hearts were dehydrated in an ethanol gradient and embedded in epoxy resin 618 (Shanghai Resin Factory, Shanghai, China). Ultrathin sections were generated from a Reichert Ultracut E ultramicrotome (Leica, Heidelberg, Germany), stained with both uranyl acetate and lead citrate for $1 \mathrm{~h}$, and examined under a Philips CM120 electron microscope (Royal Dutch Philips Electronics Ltd, Amsterdam, the Netherlands) at $60 \mathrm{kV}$. Autophagosomes and autolysosomes were counted in 6 sections per group, averaged and expressed as the number of autophagic structures per section.

\section{Measurement of plasma and cardiac angiotensin peptides}

The angiotensin peptide concentration was measured as previously described ${ }^{[20]}$. Blood was collected from the carotid 
artery and injected into a tube containing $125 \mathrm{mmol} / \mathrm{L}$ EDTA, $20 \mathrm{mmol} / \mathrm{L}$ phenanthroline, $0.2 \%$ neomycin, $0.1 \mathrm{mmol} / \mathrm{L} \mathrm{kal}-$ likrein, 2\% ethanol, and 2\% DMSO to prevent angiotensin breakdown. The blood was then centrifuged at $4{ }^{\circ} \mathrm{C}$. The plasma ( $250 \mu \mathrm{L}$ for each mouse) and the rinsed and blotted cardiac tissues (80 $\mathrm{mg}$ for each heart) were snap-frozen for storage at $-80^{\circ} \mathrm{C}$. Before the measurements of cardiac angiotensin peptide, cardiac tissues were homogenized in pure ethanol, evaporated and reconstituted in Tris buffer. Both plasma and cardiac angiotensin peptides were extracted using phenylsilylsilica and separated by high-performance liquid chromatography (HPLC), and angiotensin concentrations were measured by radioimmunoassay. The detection limits were $2 \mathrm{fmol} / \mathrm{mL}$ and $2 \mathrm{fmol} / \mathrm{mg}$ for the plasma and the tissue, respectively.

\section{Real-time quantitative reverse transcription PCR}

RNA was extracted from the left ventricles of the mice using TRIzol reagent (Invitrogen, Carlsbad, CA, USA). It was reverse transcribed into cDNA using a TOYOBO ReverTra Ace- $\mathrm{a}-{ }^{\circledR}$ RT-PCR kit, according to the manufacturer's instructions. A SYBR RT-PCR kit (Takara, Dalian, China) was employed for real-time quantitative PCR analysis. The PCR reaction was monitored using an ABI PRISM 7900 System (Applied Biosystems, Carlsbad, CA, USA). The primers were designed by Takara for atrial natriuretic peptide (ANP), brain natriuretic peptide (BNP), skeletal a-actin (SAA), transforming growth factor- $\beta 1$ (TGF- $\beta 1$ ), collagen type I a1 (Col1a1), collagen type III a1 (Col3a1), autophagy-related gene 5 (ATG5), autophagy-related 16-like 1 (ATG16 L1) and glyceraldehyde3-phosphate dehydrogenase (GAPDH) (Table 1). A comparative $\mathrm{Ct}$ method was used to determine the expression levels of the genes in question.

\section{Western blot analysis}

Total proteins extracted from the left ventricles of the mice were size-fractionated by SDS-PAGE and transferred to Immobilon-P membranes (Millipore) as previously described $^{[21]}$. The blotted membranes were incubated with antibodies against phosphorylated protein kinase $\mathrm{C}$ (pPKC) a, pPKC $\beta I$, pPKC $\beta I I$ (1:300, Santa Cruz Biotechnology, Santa Cruz, CA, USA), pPKC $\gamma$, phosphorylated extracellular signalregulated kinase1/2 (pERK1/2), ERK1/2, phosphorylated Jun N-terminal kinase (pJNK), JNK, phosphorylated P38 mitogenactivated protein kinase (pP38 MAPK), P38 MAPK, Light chain 3-II (LC3-II) and Beclin-1 (1:1000; Cell Signal Technology, Danvers, MA, USA), and later with an HRP-conjugated secondary antibody (1:5000; KANGCHEN Biotechnology, China). GAPDH expression was used as an internal control. The membranes were detected using an ECL Western-blotting Detection Reagent kit (catalogue RPN2106; GE Healthcare, Fairfield, CT, USA), with the LAS-3000 detection system. Quantification of immunoreactivity was performed via densitometric analysis using ImagePro 5.0 (Media Cybernetics, Inc, Silver Spring, MD, USA).
Table 1. Primers for real-time quantitative reverse transcriptional PCR.

\begin{tabular}{ll}
\hline Gene names & \multicolumn{1}{c}{ Primer Sequence $\left(5^{\prime}-3^{\prime}\right)$} \\
\hline \multirow{2}{*}{ ANP } & Forward: TGACAGGATTGGAGCCCAGA \\
& Reverse: GACACACCACAAGGGCTTAGGA \\
BNP & Forward: GAGGTCACTCCTATCCTCTGG \\
& Reverse: GCCATTCCTCCGACTTTTCTC \\
SAA & Forward: AGCAGATGTGGATCACCAAG \\
& Reverse: CTGCAACCACAGCACGATTG \\
TGF- 31 & Forward: GTGTGGAGCAACATGTGGACTCTA \\
& Reverse: TTGGTTCAGCCACTGCCGTA \\
Col1a1 & Forward: GACATGTTCAGCTTTGTGACCTC \\
& Reverse: GGGACCCTTAGGCCATTGTGTA \\
Col3a1 & Forward: CAGGAGCCAGTGGCCATAA \\
& Reverse: TCTCGACCTGGCTGACCATC \\
ATG5 & Forward: AGATGGACAGCTGCACACACTTG \\
& Reverse: GCATTGGCTCTATCCCGTGAA \\
ATG16L1 & Forward: GCTCCCGTGATGACCTGCTAA \\
& Reverse: CTGAGCCTGCTGCCACGTAA \\
GAPDH & Forward: ACCACAGTCCATGCCATCAC \\
& Reverse: TCCACCACCCTGTTGCTGTA \\
\hline
\end{tabular}

\section{Statistical analysis}

The statistical analysis were performed using the SPSS (Statistical Package for the Social Sciences) software package, version 16.0 (SPSS Inc, Chicago, IL, USA), and the results are expressed as the mean \pm SEM. All statistical significance was determined by one-way ANOVA, followed by Tukey's test for pairs of mean values. A value of $P<0.05$ was considered statistically significant.

\section{Results}

Effect of ALK on cardiac hypertrophy, fibrosis and dysfunction

Four weeks after TAC, all experimental mice were found to have survived and subsequently received both echocardiographic and hemodynamic examinations. Both aortic blood pressure $(\mathrm{ABP})$ and left ventricle end-systolic pressure (LVESP) increased by more than 1.5-fold after TAC (Figure S1). Echocardiographic results showed increased left ventricular wall thickness and impaired cardiac function in the TAC control group, compared with those in the Sham group (Figure 1A, 1B). Moreover, an augmented HW/BW, as well as an elevated CSA of cardiomyocytes, was observed (Figure $1 C, 1 D)$. Additionally, the reprogramming of $A N P, B N P$, and $S A A$ was observed in the TAC group (Figure 1E). Although ALK did not elicit any notable effects on cardiac geometry and function at baseline, it evidently ameliorated all maladaptive responses induced by TAC (Figure 1), without lowering either ABP or LVESP (Figure S1). Masson's trichrome staining showed a markedly elevated interstitial collagen volume in the TAC control group as compared with that in the Sham group. However, the extracellular matrix change was significantly inhibited in the TAC-ALK group (Figure 1F). The subsequent analysis of mRNA levels of TGF- $\beta 1$, Col1a1, and Col3a1 also demonstrated elevated responses in the TAC control group 
A

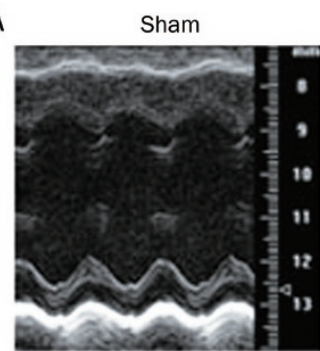

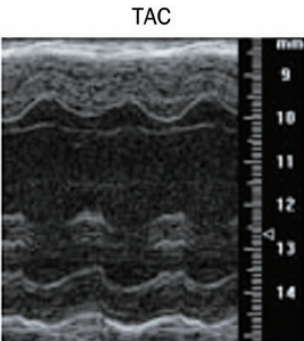
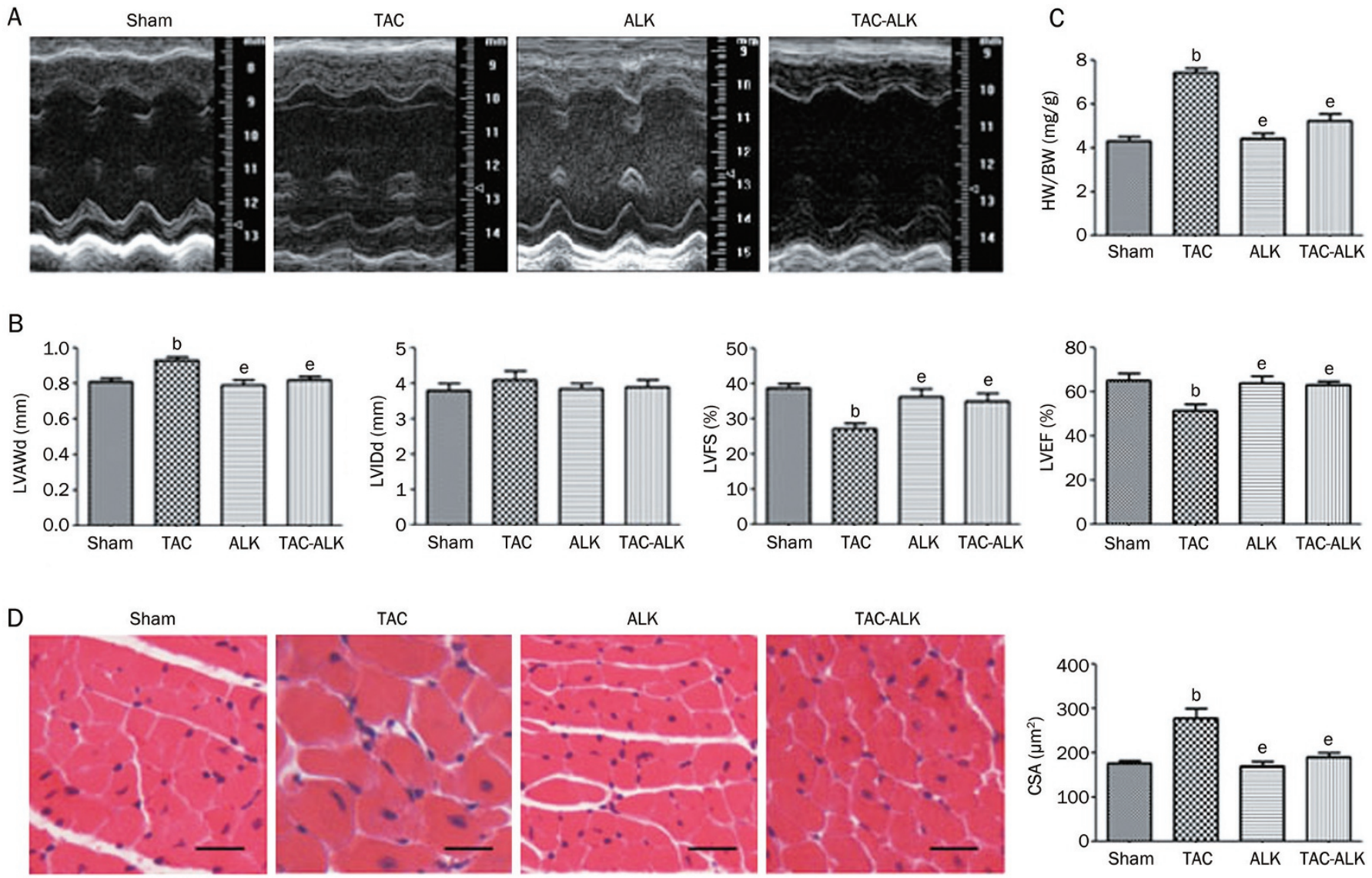

E

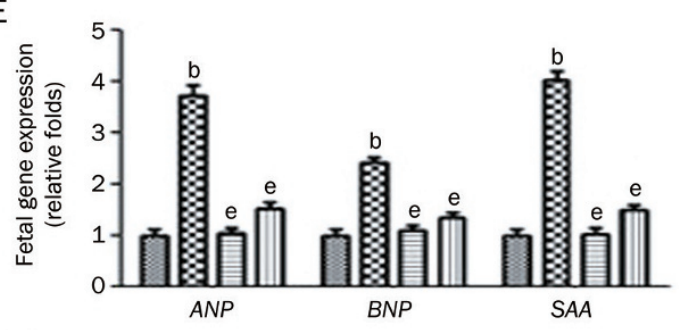

G

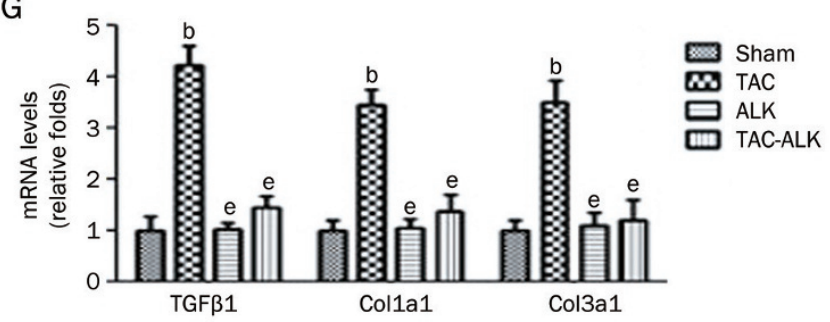

ALK

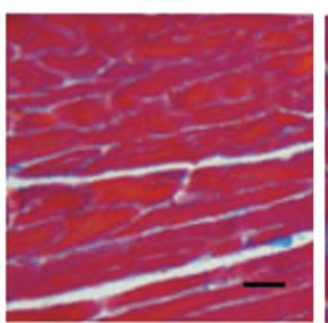

TAC

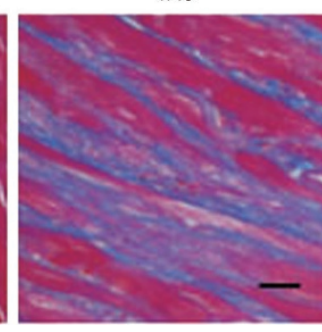

Sham

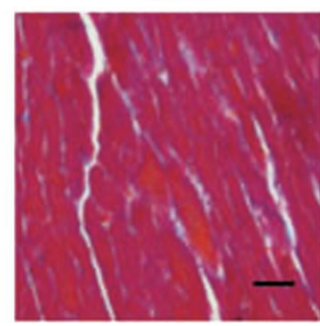

Figure 1. Effect of ALK on TAC-induced cardiac hypertrophy, fibrosis and dysfunction 4 weeks after TAC. (A) Representative M-Mode images. (B) Echocardiographic parameter analysis. LVAWd, left ventricular anterior wall thickness at end-diastole; LVIDd, left ventricular internal dimension at enddiastole; LVFS, left ventricular fractional shortening; LVEF, left ventricular ejection fraction. (C) Heart to body weight ratio (HW/BW). (D) Representative HE-stained left ventricular sections (scale bar: $20 \mu \mathrm{m}$ ) and quantitative analysis of cross-sectional area (CSA). (E) The expression of hypertrophyassociated genes. Bars indicate the relative folds of the expression of ANP, BNP, and SAA of the internal control. GAPDH served as the internal control. ANP, atrial natriuretic peptide. BNP, brain natriuretic peptide. SAA, skeletal $\alpha$-actin. (F) Representative Masson's trichrome-stained left ventricular sections (scale bar: $20 \mu \mathrm{m}$ ) and fibrotic area analysis. Blue areas indicate fibrotic staining. (G) The expression of fibrosis-associated mRNA. GAPDH was used as the internal control. TGF $\beta 1$, transforming growth factor $\beta 1$. Col1a1, collagen type I $\alpha 1$. Col3a1, collagen type III $\alpha 1$. $n=6$. Mean \pm SEM. ${ }^{\mathrm{b}} \mathrm{P}<0.05$ vs Sham. ${ }^{\mathrm{e}} \mathrm{P}<0.05$ vs TAC. ALK, aliskiren $\left(150 \mathrm{mg} \cdot \mathrm{kg}^{-1} \cdot \mathrm{d}^{-1}, \mathrm{po}\right)$ 
as compared with those in the Sham group, but each of these enhanced fibrotic markers decreased significantly in the TACALK group (Figure 1G).

\section{Effect of ALK on cardiac autophagy}

Electron microscopic analysis showed that the number of autophagic structures increased significantly in the TAC control group compared with the Sham group. ALK did not change the number of autophagic structures at baseline. However, ALK clearly ameliorated the level of autophagy when its effects were examined 4 weeks after TAC (Figure 2A, 2B). Corresponding mRNA expression levels of autophagy-related markers, such as Atg5 and Atg16 L1, were notably upregulated in the TAC control group, but were distinctly downregulated following ALK administration (Figure 2C). Further experiments demonstrated that ALK overtly diminished TACinduced elevation in protein expression levels of both LC3-II and Beclin-1 (Figure 2D).

Effect of autophagy inhibition on TAC-induced cardiac hypertrophy, fibrosis and dysfunction

To evaluate the role of autophagy in TAC-induced cardiac hypertrophy, fibrosis and dysfunction, Sham and TAC mice were treated with the autophagy inhibitor, 3-MA, for 4 weeks prior to the assessment of cardiac geometry and function. Our data revealed that 4 weeks of TAC without 3-MA treatment induced significant cardiac hypertrophy, fibrosis and dysfunction. Although 3-MA itself did not affect cardiac morphology or function at baseline, it notably attenuated TAC-induced cardiac hypertrophy, fibrosis and dysfunction, as evidenced by the overtly decreased LVAWd, HW/BW, CSA, and interstitial collagen volume, as well as the improved LVFS and LVEF (Figure 3), without lowering either ABP or LVESP (Figure S2). These data suggested a role for autophagy in TAC-induced cardiac hypertrophy, fibrosis and dysfunction.

Effect of ALK on plasma and cardiac levels of angiotensin, phosphorylation of cardiac PKC isoforms and mitogen-activated protein kinase

The plasma and cardiac levels of Ang I and Ang II were significantly elevated in the TAC control group, compared with those in the Sham group. ALK effectively downregulated the TAC-induced upregulation of both Ang I and Ang II (Figure 4A). To investigate which PKC isoforms contributed to TAC-
A
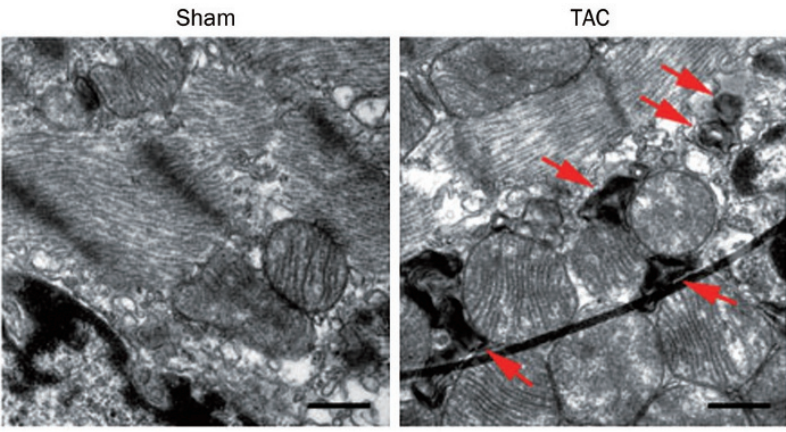

ALK

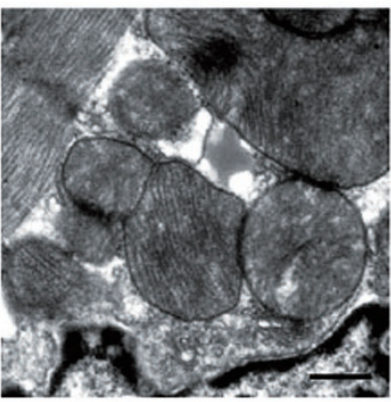

TAC-ALK

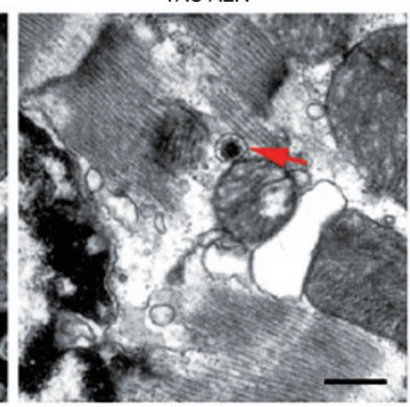

B

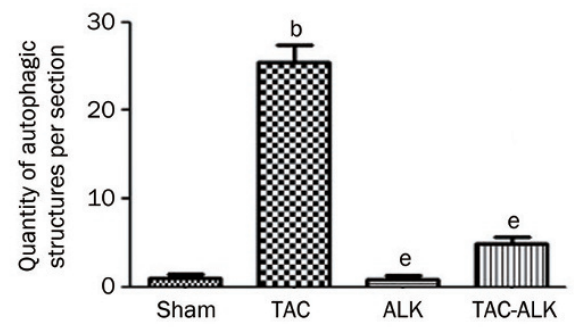

C

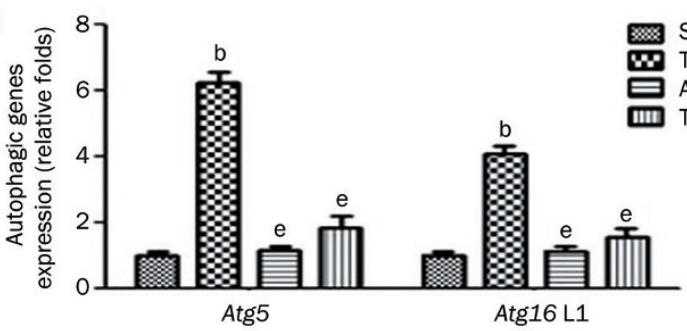

D
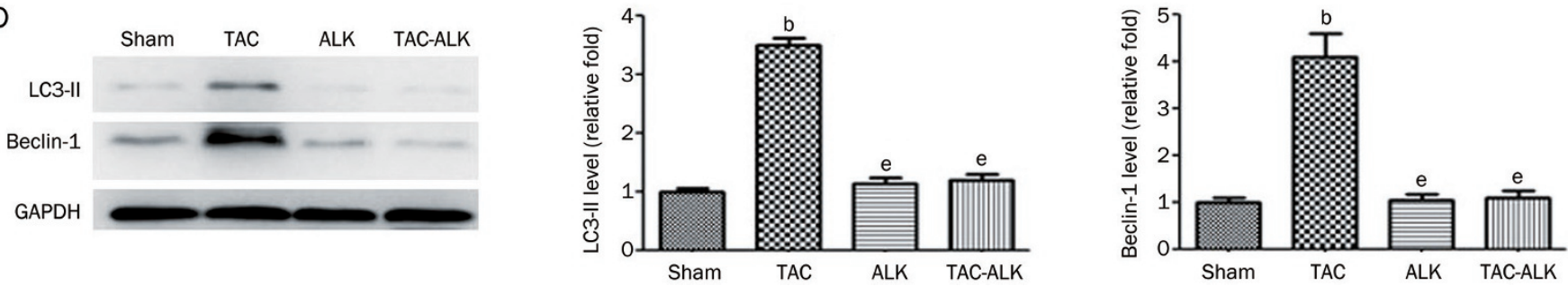

Figure 2. Effect of ALK on TAC-induced change in autophagy. (A) Representative TEM images of left ventricular sections (scale bar: 500 nm, arrows indicating autophagic structures). (B) Quantitative analysis of autophagic structures. (C) Quantitative analysis of autophagic genes. (D) Representative gel blots and quantitative analysis of LC3-II and Beclin-1. $n=6$. Mean \pm SEM. ${ }^{\mathrm{b}} P<0.05$ vs Sham. ${ }^{\mathrm{e}} P<0.05$ vs TAC. 
A

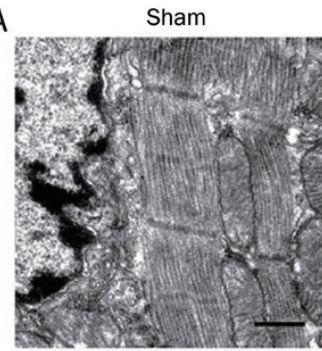

B
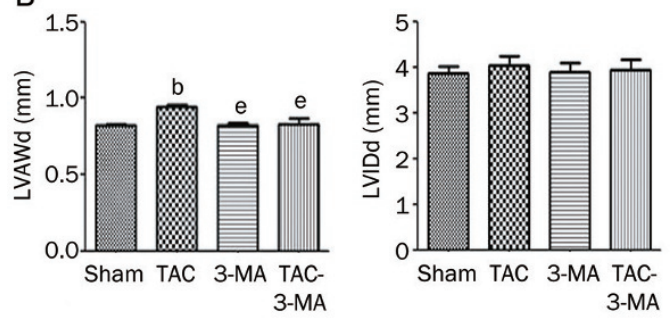

$\mathrm{D}$
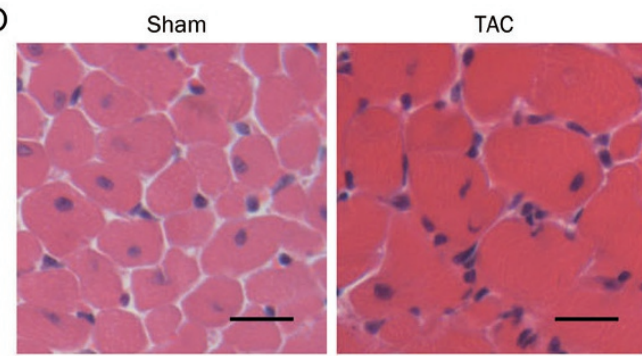

E

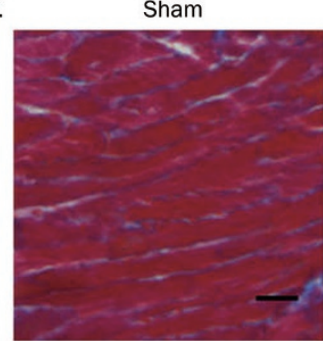

TAC
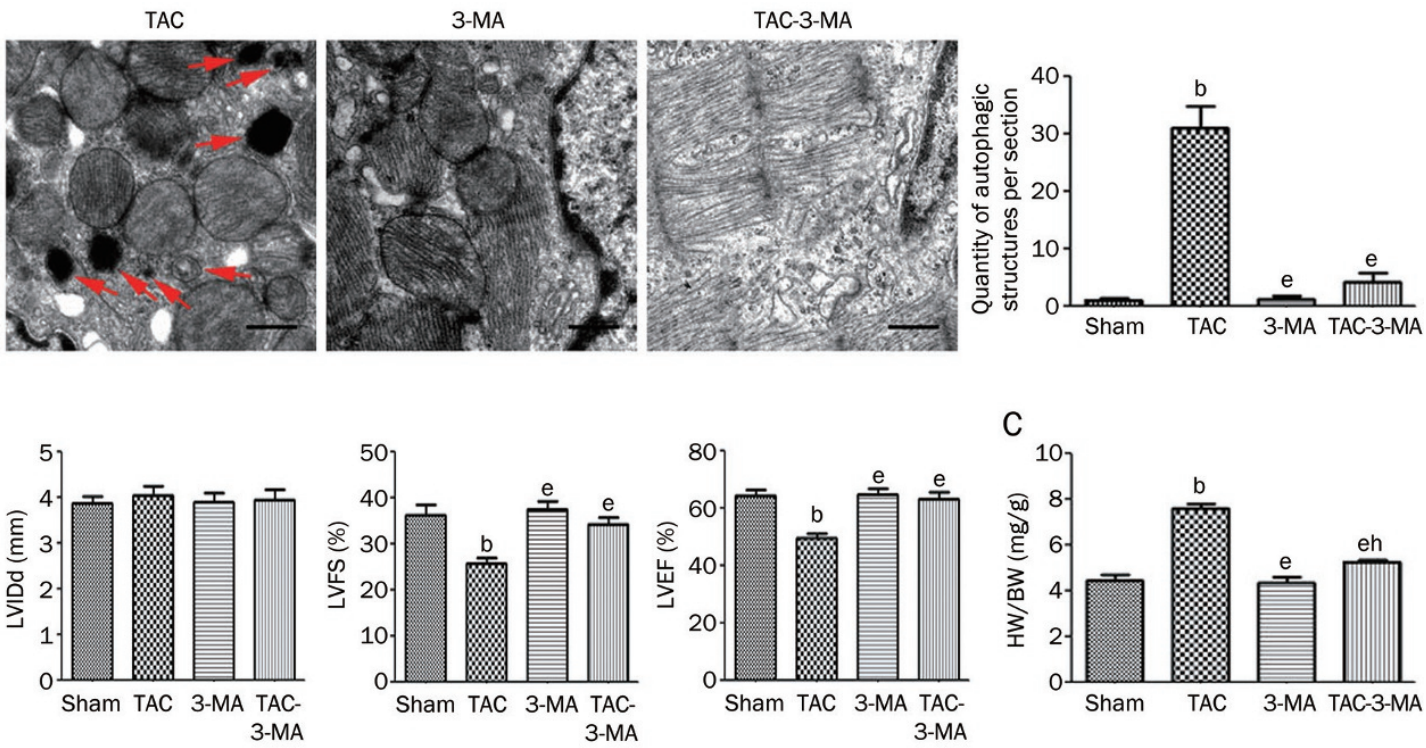

C
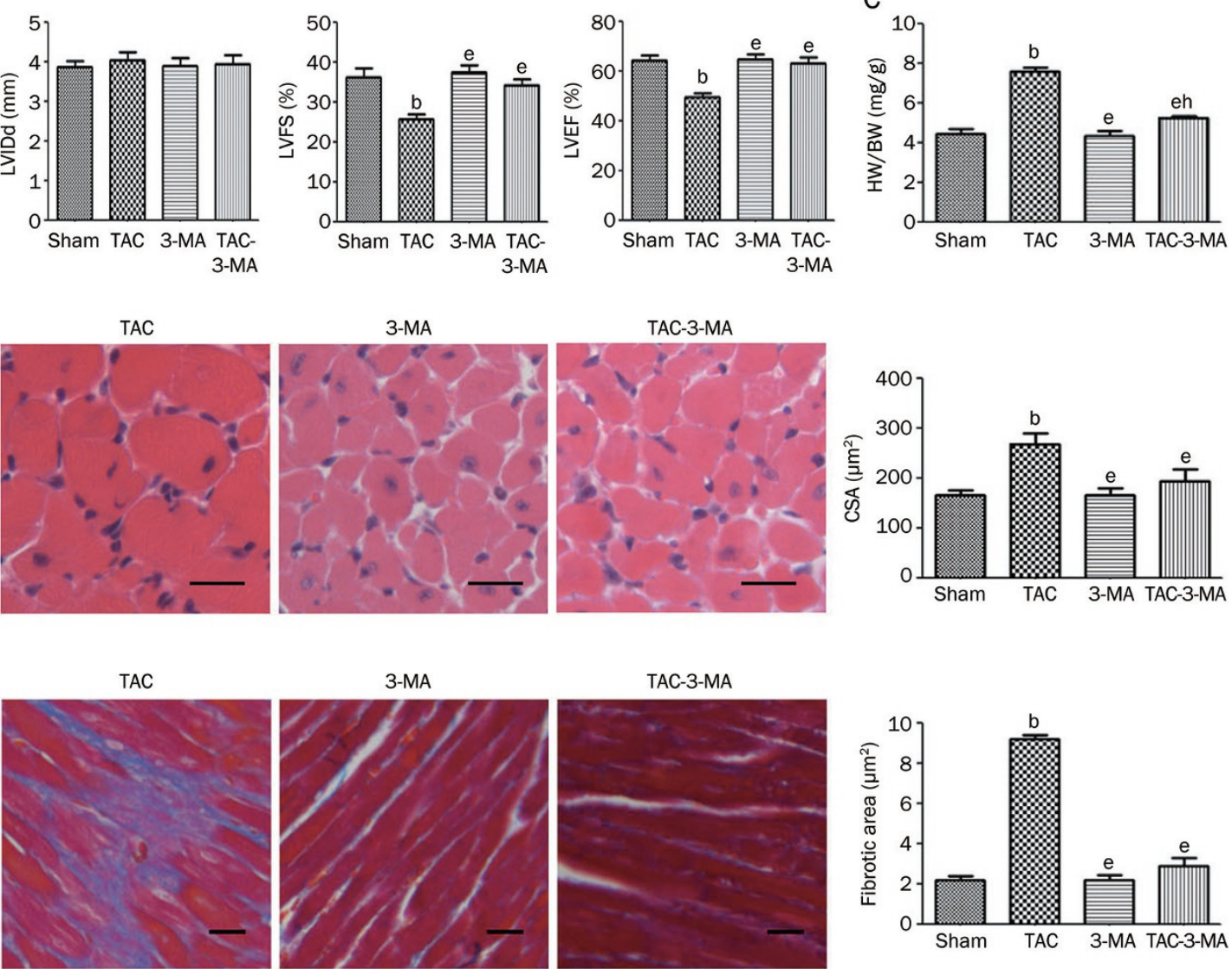

3-MA

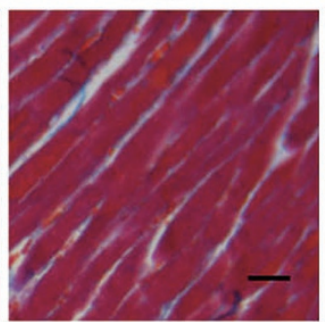

TAC-3-MA

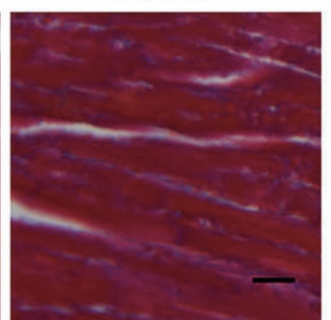



Figure 3. Effect of 3-MA on TAC-induced cardiac hypertrophy, fibrosis and dysfunction. (A) Representative TEM images of left ventricular sections (scale bar: $500 \mathrm{~nm}$, arrows indicating autophagic structures). (B) Echocardiographic parameter analysis. LVAWd, left ventricular anterior wall thickness at end-diastole; LVIDd, left ventricular internal dimension at end-diastole; LVFS, left ventricular fraction shortening; LVEF, left ventricular eject fraction. (C) Heart to body weight ratio (HW/BW). (D) Representative HE-stained left ventricular sections (scale bar: $20 \mu \mathrm{m}$ ) and quantitative analysis of crosssectional area (CSA). (E) Representative Masson's trichrome-stained left ventricular sections (scale bar: $20 \mu \mathrm{m}$ ) and quantitative analysis of fibrotic area. Blue areas indicate fibrotic staining. $n=6$. Mean \pm SEM. ${ }^{b} P<0.05$ vs Sham. ${ }^{e} P<0.05$ vs TAC. ${ }^{\text {h }} P<0.05$ vs 3-MA. 3-MA, 3-methyladenine (autophagy inhibitor, $10 \mathrm{mg} \cdot \mathrm{kg}^{-1}$ per week, ip).

induced cardiac abnormalities and whether these activated PKC isoforms may be inhibited by ALK, the expression levels of pPKCa, pPKC $\beta \mathrm{I}, \mathrm{pPKC} \beta \mathrm{II}$, and $\mathrm{pPKC} \gamma$ were examined. The isoforms $\mathrm{pPKC} \beta \mathrm{II}$ and $\mathrm{pPKC} \gamma$ did not increase, but $\mathrm{pPKCa}$ and pPKC $\beta I$ both increased in the TAC control group. Of note, the elevated cardiac isoforms pPKCa and pPKC $\beta$ I were apparently attenuated in response to chronic renin inhibition with ALK (Figure 4B). To explore the molecular mechanism of this process further, the mitogen-activated protein kinases (MAPK) were investigated. Pressure overload induced an evi- dent rise in the phosphorylation levels of ERK1/2, p38 MAPK, and JNK1/2; however, ALK treatment markedly blocked the activation of ERK1/2, whereas p38 MAPK and JNK1/2 were not significantly affected (Figure $4 \mathrm{C}$ ).

\section{PKC $\beta I / \alpha$, ERK1/2, and autophagy}

To test whether PKC isoforms participate in TAC-induced autophagy, mechanically stretched-cardiomyocytes were pretreated with a PKC $\beta \mathrm{I} / \mathrm{\alpha}$ inhibitor. As indicated by the results of the Western blot, mechanical stress significantly enhanced 

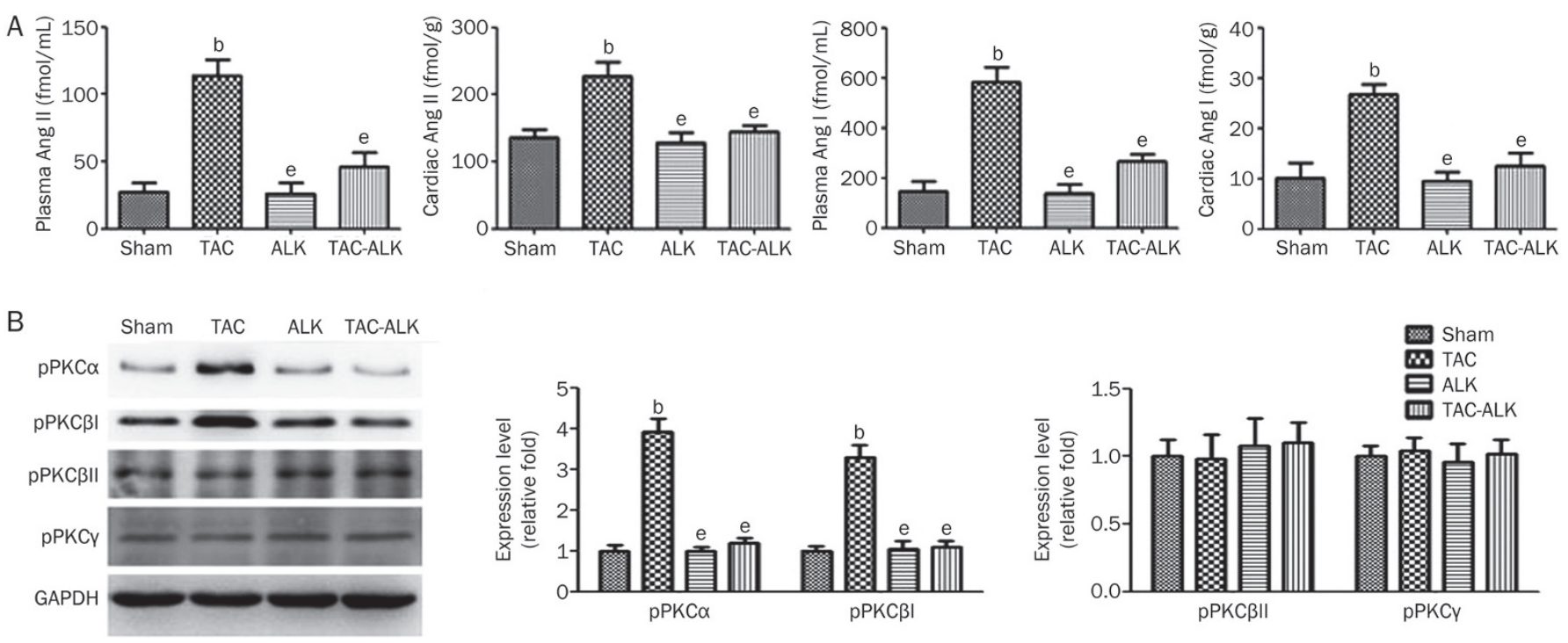

C
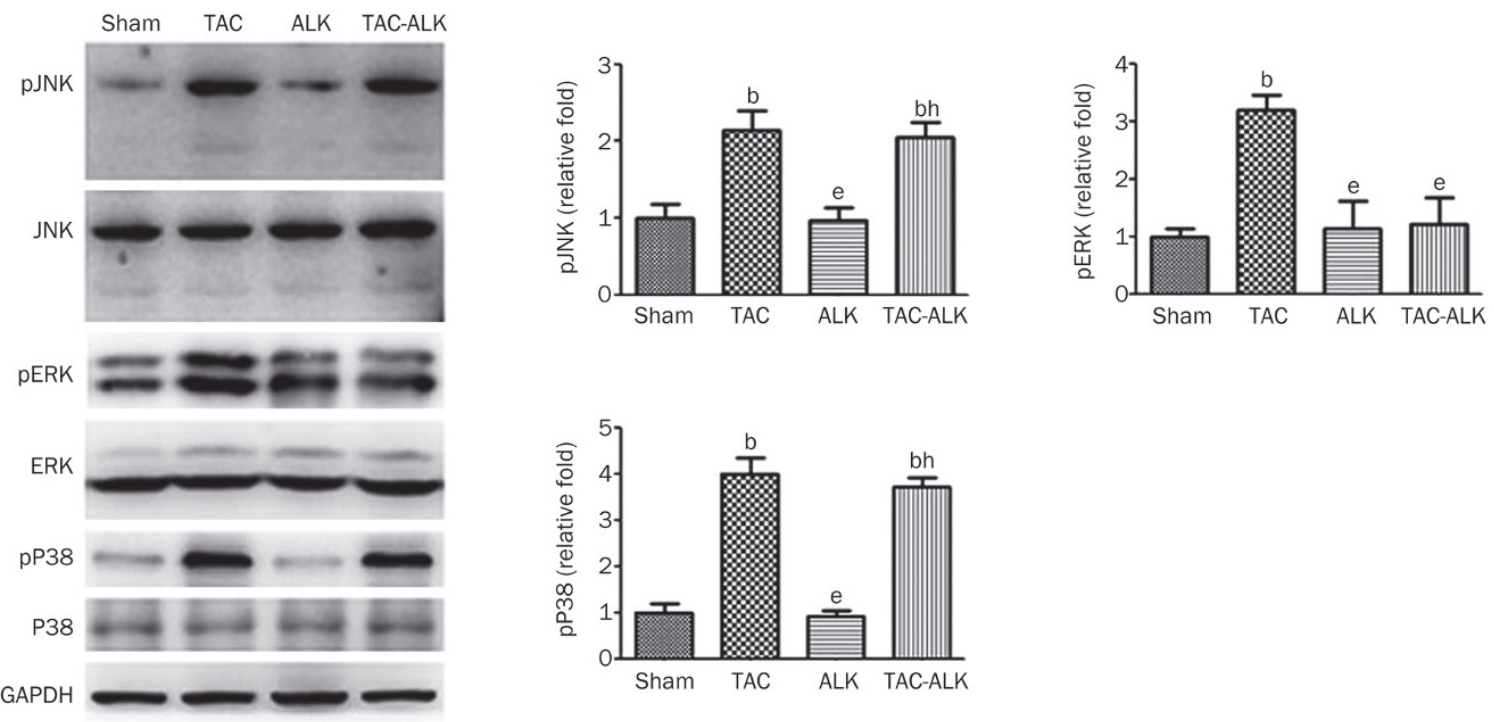

Figure 4. Effect of ALK on TAC-induced change in the levels of Ang I/Ang II, PKC isoforms, and MAPK. (A) Angiotensin concentrations in plasma and heart of experimental mice. Ang I, Angiotensin I; Ang II, Angiotensin II. (B) Representative gel blots and quantitative analysis of phosphorylated PKC $\alpha$, $\beta I, \beta I I$, and $\gamma$. (C) Representative gel blots of ERK, pERK, p38, pP38, JNK, and pJNK, and quantitative analysis of pERK, pP38, and pJNK. GAPDH served as the internal control. $n=6$. Mean \pm SEM. ${ }^{b} P<0.05$ vs Sham. ${ }^{\mathrm{e}} P<0.05$ vs TAC. ${ }^{\text {h }} P<0.05$ vs ALK.

the levels of pERK1/2 and autophagic flux, an outcome that was prevented by pretreatment with the PKC $\beta \mathrm{I}$ inhibitor, CGP53353, but not the PKCa inhibitor, Ro-31-0432 (Figure $5 \mathrm{~A}, 5 \mathrm{~B})$. To examine the effect of ERK inhibition on cardiomyocyte autophagy, cardiomyocytes were pretreated with the ERK inhibitor, U0126. Our data revealed that U0126 notably attenuated mechanical stress-induced elevations in LC3-II and Beclin-1 (Figure 5C). Taken together, our in vitro results revealed that mechanical stress-induced autophagy was partly mediated by the PKC $\beta I$-ERK1/ 2 dependent pathway.

To confirm the relationship among PKC $\beta$ I, ERK1/2, and autophagy in vivo, a PKC $\beta$ I inhibitor, LY333531 (LY), was employed. Echocardiographic and histological analysis showed that LY effectively inhibited cardiac hypertrophy, fibrosis and dysfunction induced by TAC without lowering ABP and LVESP (Figure S3, S4). Further electron microscopic analysis showed that the number of autophagic structures decreased significantly following LY administration when compared with the amount of structures noted in the TAC control group (Figure 5D, 5E), and the same results were observed in the corresponding mRNA expression levels of autophagyrelated markers Atg5 and Atg16 L1 (Figure 5F). Moreover, Western blot results demonstrated that LY also clearly abrogated the elevations in pERK1/2, LC3-II, and Beclin-1 induced by TAC (Figure 5G). Taken together, our results consistently demonstrated the close relationship among PKC $\beta$ I, ERK1/2, and autophagy. 

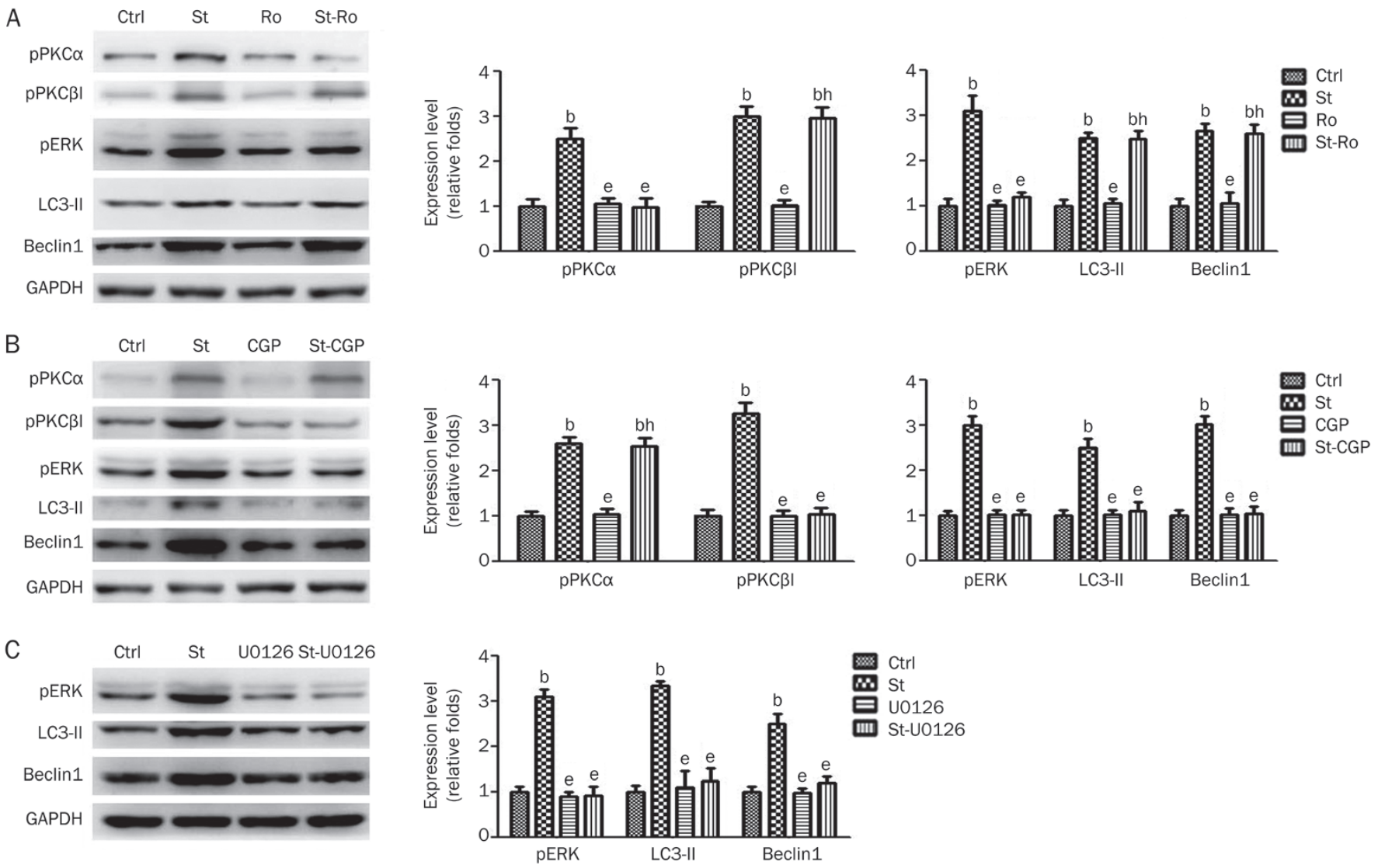

D
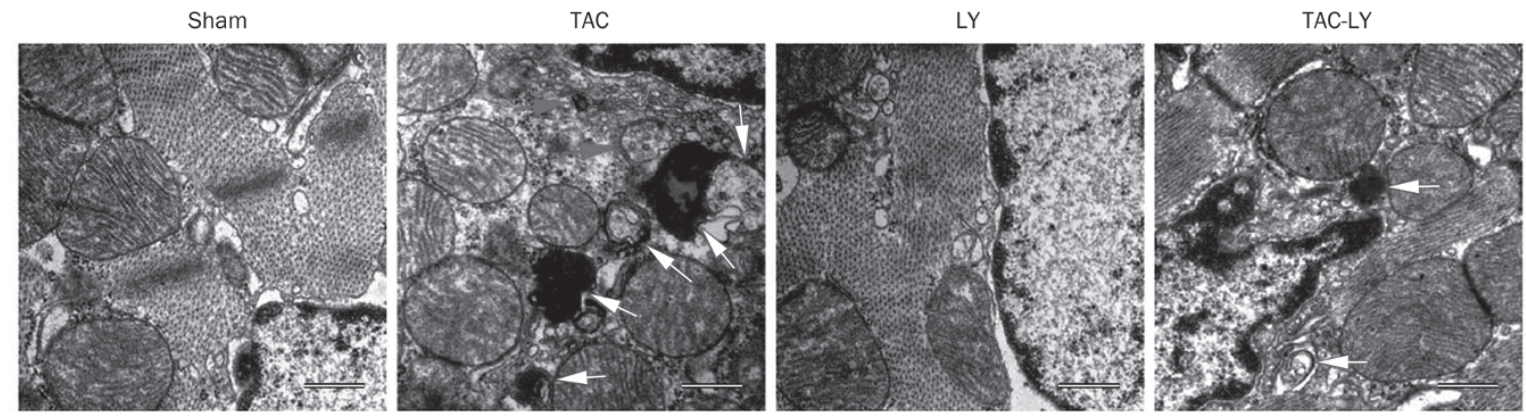

E
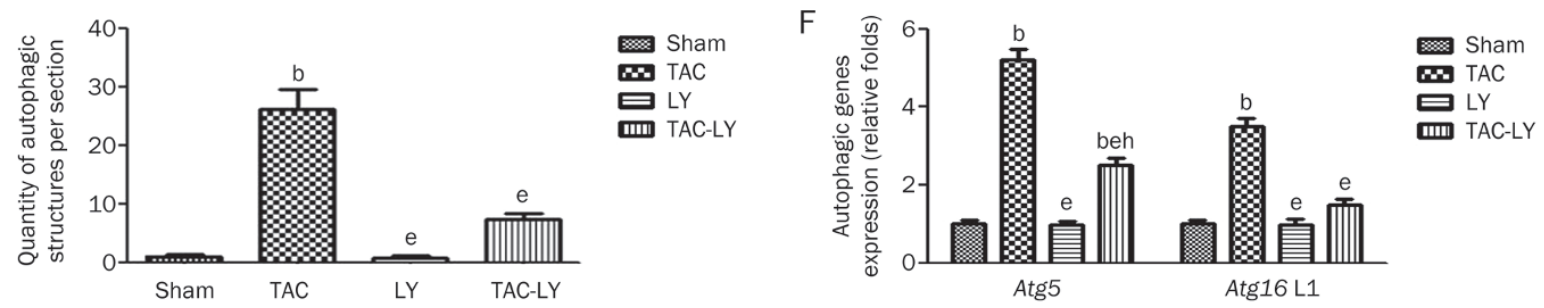

Figure 5A-5F. Relationship among PKC $\beta \mathrm{I} / \alpha$, ERK, and autophagy. (A) Relationship among PKC $\alpha, p E R K 1 / 2$ and autophagy. Representative gel blots and quantitative analysis of $\mathrm{pPKC} \alpha, \mathrm{pPKC} \beta \mathrm{I}, \mathrm{pERK}, \mathrm{LC} 3-\mathrm{Il}$, and Beclin-1 are shown. Four parallel experiments were indicated. Mean \pm SEM. ${ }^{\mathrm{b}} \mathrm{P}<0.05 \mathrm{vs}$ Ctrl. ${ }^{\mathrm{e}} \mathrm{P}<0.05$ vs St. ${ }^{\mathrm{h}} \mathrm{P}<0.05$ vs Ro. St, Stretch by $20 \%$ for 24 h. Ro, Ro-31-0432 (a PKC $\alpha$ inhibitor, 200 pmol/mL). (B) Relationship among PKC 3 , pERK1/2, and autophagy. Representative gel blots and quantitative analysis of pPKC $\alpha, p P K C \beta I, p E R K$, LC3-II, and Beclin-1 are shown. Four parallel experiments were indicated. Mean \pm SEM. ${ }^{b} P<0.05$ vs Ctrl. ${ }^{e} P<0.05$ vs St. ${ }^{h} P<0.05$ vs CGP. St, Stretch by $20 \%$ for 24 h. CGP, CGP53353 (a PKC $\beta$ I inhibitor, $6.0 \mathrm{nmol} / \mathrm{mL}$ ). (C) Relationship between pERK1/2 and autophagy. Representative gel blots and quantitative analysis of pERK, LC3-II and Beclin-1 are shown. Four parallel experiments were indicated. Mean \pm SEM. ${ }^{\mathrm{b}} P<0.05$ vs Ctrl. ${ }^{\mathrm{e}} P<0.05$ vs St. ${ }^{\mathrm{h}} P<0.05$ vs U0126. St, Stretch by $20 \%$ for $24 \mathrm{~h}$. U0126, an ERK inhibitor (10 $\mu \mathrm{mol} / \mathrm{L})$. (D) TEM images and (E) quantitative analysis of autophagic structures on cardiac tissue sections, scale bar: $500 \mathrm{~nm}$, arrows indicating autophagic structures. (F) Quantitative analysis of autophagic genes ATG5 and ATG16 L1. 

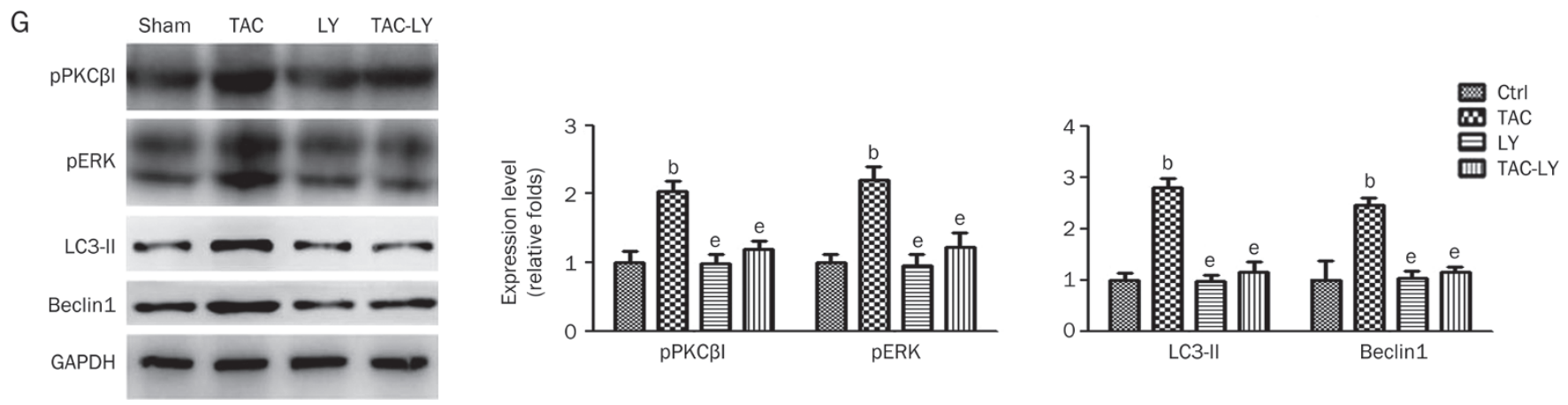

Figure 5G. (G) Representative gel blots and quantitative analysis of $p P K C \beta I, p E R K 1 / 2$, LC3-II, and Beclin-1. $n=6$. Mean \pm SEM. ${ }^{b} P<0.05$ vs Sham. ${ }^{\mathrm{e}} P<0.05$ vs TAC. ${ }^{\mathrm{h}} P<0.05$ vs LY. LY, LY333531 (a PKC 31 inhibitor, $1 \mathrm{mg} \cdot \mathrm{kg}^{-1} \cdot \mathrm{d}^{-1}, p o$ ).

\section{Discussion}

In the current study, we explored the effect of ALK monotherapy on the development of cardiac hypertrophy and fibrosis in mice subjected to a 4-week period of hemodynamic stress. The findings of our study showed that ALK attenuated pressure overload-induced cardiac hypertrophy, fibrosis and insufficiency, independent of BP reduction.

To investigate the inhibitory mechanisms of ALK, autophagy was first assessed. The role of autophagy in cardiac disorders is complex. In high-fat diet-induced obesity, it was reported that rescuing the autophagosome maturation process via Akt2 knockout was beneficial to both cardiac geometry and function ${ }^{[22]}$. In the pressure overload-induced hypertrophic heart, there still exist different opinions. Some research revealed that activating autophagy may attenuate pressure overload-induced cardiac hypertrophic responses ${ }^{[23,24]}$ whereas other studies recognized excessive autophagy as a detrimental factor in pressure overload-induced heart failure ${ }^{[12,13,25]}$. To date, a consensus has been reached that autophagy may be either adaptive or maladaptive depending on the context in which it occurs and the extent of its stimulation ${ }^{[26]}$. Our results showed a significant rise in the autophagic activity of myocardial tissues in TAC mice. ALK prevented TAC-induced cardiac geometric and functional abnormalities in part through the amelioration of TAC-induced autophagy, based on the results demonstrating that ALK treatment decreased TACinduced autophagic activity to almost its baseline level, and the inhibition of autophagic flux by 3-MA proved salutary in preserving optimal cardiac geometry and function in TAC mice. To the best of our knowledge, this is the first report on the relationship between a renin inhibitor and autophagy.

To investigate these mechanisms further, expression levels of both plasma and cardiac angiotensin, as well as cardiac phosphorylated PKC isoforms, were examined. We found that raised plasma and cardiac levels of both Ang I and Ang II were significantly negated by ALK treatment. Furthermore, the elevated phosphorylation of PKCa in the TAC control group was clearly reduced following ALK treatment. As for the PKC $\beta$, PKC $\beta I$, but not PKC $\beta I I$, was involved in TACinduced hypertrophy. ALK evidently abrogated the upregulation of PKC $\beta$ I phosphorylation, which may be of much greater importance compared with pPKCa reduction, as many previous studies indicate that a change in mouse PKC $\beta$ correlates more strongly with a corresponding change in humans ${ }^{[27,28]}$.

As ALK was found to downregulate PKC $\beta I /$ a phosphorylation and autophagy, it was postulated that TAC-induced autophagy may be a PKC $\beta \mathrm{I} / \alpha$-dependent response, and ALK may ameliorate the excessive autophagy via a PKC $\beta \mathrm{I} /$ a-dependent pathway. The relationship between PKC $\beta \mathrm{I} / \mathrm{a}$ and autophagy was first investigated in vitro. It was intriguing that the mechanical stress-induced elevations of LC3-II and Beclin-1 were diminished by the treatment with a PKC $\beta \mathrm{I}$ inhibitor, as opposed the use of a PKCa inhibitor. Additionally, the ERK1/2 inhibitor, U0126, notably reduced mechanical stress-induced autophagy. The relationship among pPKC $\beta \mathrm{I}$, pERK1/2 and autophagy was further confirmed in vivo. Taken together, it may be inferred that ALK protects against TACinduced cardiac hypertrophy, fibrosis and dysfunction, in part via the reduction of PKC $\beta$ I-ERK1/2-regulated autophagy.

It has previously been reported that ALK may increase cardiac bradykinin levels, which may contribute to its cardioprotective effects in the (mRen-2)27 $\mathrm{rat}^{[29]}$. In the current study, however, we demonstrated that ALK exhibits a cardioprotective effect by modulating excessive autophagic activity, which represents a novel idea.

As our data suggested, ALK monotherapy may inhibit the development of cardiac hypertrophy and fibrosis, independent of BP reduction, in the setting of 4 weeks of pressure overload in mice, by blocking Ang II-PKC 3 I-ERK1/2-regulated autophagy. This study provides insight into the pathophysiology of cardiac hypertrophy and fibrosis, as well as the mechanism of the inhibitory effects of ALK.

\section{Acknowledgements}

We would like to thank Mr Guo-ping ZHANG at the Institute of Biomedical Science, Fudan University for his technical assistance. This work was supported by grants from the Key Program of National Natural Science Foundation of China (№ 30930043), the Key International (Regional) Joint Research Program of National Natural Science Foundation of China (No 81220108003), and the Key Basic Research Program of Shanghai (No 11JC1402400), which were awarded to Yun- 
zeng ZOU. Meanwhile, it was supported by the grant from Natural Science Foundation of Zhejiang Province of China (№ LQ14H020002), which was awarded to Wen-bin ZHANG.

\section{Author contribution}

Li-qing WENG and Yun-zeng ZOU designed the research; Liqing WENG, Wen-bin ZHANG, Yong YE, Pei-pei YIN, and Jie YUAN performed the research; Xing-xu WANG, Le KANG, Sha-sha JIANG, Jie-yun YOU, and Jian WU analyzed data; Liqing WENG, Wen-bin ZHANG, Yong YE, Pei-pei YIN, and Jie YUAN wrote the paper; Hui GONG, Jun-bo GE, and Yun-zeng ZOU revised the manuscript.

\section{Supplementary information}

Supplementary information is available at the APS web site.

\section{References}

1 Frohlich ED, Apstein C, Chobanian AV, Devereux RB, Dustan HP, Dzau V, et al. The heart in hypertension. N Engl J Med 1992; 327: 9981008.

2 Dahlof B, Devereux RB, Kjeldsen SE, Julius S, Beevers G, de Faire $\mathrm{U}$, et al. Cardiovascular morbidity and mortality in the Losartan Intervention For Endpoint reduction in hypertension study (LIFE): a randomised trial against atenolol. Lancet 2002; 359: 995-1003.

3 de Simone G, Gottdiener JS, Chinali M, Maurer MS. Left ventricular mass predicts heart failure not related to previous myocardial infarction: the Cardiovascular Health Study. Eur Heart J 2008; 29 : 741-7.

4 Roger VL, Go AS, Lloyd-Jones DM, Benjamin EJ, Berry JD, Borden WB, et al. Heart disease and stroke statistics - 2012 update: a report from the American Heart Association. Circulation 2012; 125: e2e220.

5 Frey N, Olson EN. Cardiac hypertrophy: the good, the bad, and the ugly. Annu Rev Physiol 2003; 65: 45-79.

6 Kjeldsen SE, Dahlof B, Devereux RB, Julius S, Aurup P, Edelman J, et al. Effects of losartan on cardiovascular morbidity and mortality in patients with isolated systolic hypertension and left ventricular hypertrophy: a Losartan Intervention for Endpoint Reduction (LIFE) substudy. JAMA 2002; 288: 1491-8.

7 Lang CC, Struthers AD. Targeting the renin-angiotensin-aldosterone system in heart failure. Nat Rev Cardiol 2013; 10: 125-34.

8 Siragy HM. Rationale for combining a direct renin inhibitor with other renin-angiotensin system blockers. Focus on aliskiren and combinations. Cardiovasc Drugs Ther 2011; 25: 87-97.

9 Shah AM, Shin SH, Takeuchi M, Skali H, Desai AS, Kober L, et al. Left ventricular systolic and diastolic function, remodelling, and clinical outcomes among patients with diabetes following myocardial infarction and the influence of direct renin inhibition with aliskiren. Eur J Heart Failure 2012; 14: 185-92.

10 Harel Z, Gilbert C, Wald R, Bell C, Perl J, Juurlink D, et al. The effect of combination treatment with aliskiren and blockers of the reninangiotensin system on hyperkalaemia and acute kidney injury: systematic review and meta-analysis. BMJ 2012; 344: e42.

11 Angeli F, Reboldi G, Mazzotta G, Poltronieri C, Garofoli M, Ramundo E, et al. Safety and efficacy of aliskiren in the treatment of hypertension and associated clinical conditions. Curr Drug Saf 2012; 7: 76-85.
12 Zhu H, Tannous P, Johnstone JL, Kong Y, Shelton JM, Richardson JA, et al. Cardiac autophagy is a maladaptive response to hemodynamic stress. J Clin Invest 2007; 117: 1782-93.

13 Rothermel BA, Hill JA. Autophagy in load-induced heart disease. Circ Res 2008; 103: 1363-9.

14 Steinberg SF. Cardiac actions of protein kinase C isoforms. Physiology (Bethesda) 2012; 27: 130-9.

15 Liu Q, Molkentin JD. Protein kinase Calpha as a heart failure therapeutic target. J Mol Cell Cardiol 2011; 51: 474-8.

16 Hambleton M, Hahn H, Pleger ST, Kuhn MC, Klevitsky R, Carr AN, et al. Pharmacological- and gene therapy-based inhibition of protein kinase Calpha/beta enhances cardiac contractility and attenuates heart failure. Circulation 2006; 114: 574-82.

17 Roman BB, Geenen DL, Leitges M, Buttrick PM. PKC-beta is not necessary for cardiac hypertrophy. Am J Physiol Heart Circ Physiol 2001; 280: H2264-70.

18 Zou Y, Akazawa H, Qin Y, Sano M, Takano H, Minamino T, et al. Mechanical stress activates angiotensin II type 1 receptor without the involvement of angiotensin II. Nat Cell Biol 2004; 6: 499-506.

19 Sano M, Minamino T, Toko H, Miyauchi H, Orimo M, Qin Y, et al. p53induced inhibition of Hif-1 causes cardiac dysfunction during pressure overload. Nature 2007; 446: 444-8.

20 Akers WS, Cross A, Speth R, Dwoskin LP, Cassis LA. Renin-angiotensin system and sympathetic nervous system in cardiac pressure-overload hypertrophy. Am J Physiol Heart Circ Physiol 2000; 279: H2797-806.

21 Zhang WB, Du QJ, Li H, Sun AJ, Qiu ZH, Wu CN, et al. The therapeutic effect of rosuvastatin on cardiac remodelling from hypertrophy to fibrosis during the end-stage hypertension in rats. J Cell Mol Med 2012; 16: 2227-37.

22 Xu X, Hua Y, Nair S, Zhang Y, Ren J. Akt2 knockout preserves cardiac function in high-fat diet-induced obesity by rescuing cardiac autophagosome maturation. J Mol Cell Biol 2013; 5: 61-3.

23 McMullen JR, Sherwood MC, Tarnavski O, Zhang L, Dorfman AL, Shioi $\mathrm{T}$, et al. Inhibition of mTOR signaling with rapamycin regresses established cardiac hypertrophy induced by pressure overload. Circulation 2004; 109: 3050-5.

$24 \mathrm{Xu} \mathrm{X,} \mathrm{Hua} \mathrm{Y,} \mathrm{Nair} \mathrm{S,} \mathrm{Bucala} \mathrm{R,} \mathrm{Ren} \mathrm{J.} \mathrm{Macrophage} \mathrm{migration} \mathrm{inhibitory}$ factor deletion exacerbates pressure overload-induced cardiac hypertrophy through mitigating autophagy. Hypertension 2014; 63: 490-9.

25 Cao DJ, Wang ZV, Battiprolu PK, Jiang N, Morales CR, Kong Y, et al. Histone deacetylase (HDAC) inhibitors attenuate cardiac hypertrophy by suppressing autophagy. Proc Natl Acad Sci U S A 2011; 108: 4123-8.

26 Nemchenko A, Chiong M, Turer A, Lavandero S, Hill JA. Autophagy as a therapeutic target in cardiovascular disease. J Mol Cell Cardiol 2011; 51: 584-93.

27 Simonis G, Briem SK, Schoen SP, Bock M, Marquetant R, Strasser $\mathrm{RH}$. Protein kinase $\mathrm{C}$ in the human heart: differential regulation of the isoforms in aortic stenosis or dilated cardiomyopathy. Mol Cell Biochem 2007; 305: 103-11.

28 Braz JC, Gregory K, Pathak A, Zhao W, Sahin B, Klevitsky R, et al. $\mathrm{PKC}$-alpha regulates cardiac contractility and propensity toward heart failure. Nat Med 2004; 10: 248-54.

29 Campbell DJ, Zhang Y, Kelly DJ, Gilbert RE, McCarthy DJ, Shi W, et al. Aliskiren increases bradykinin and tissue kallikrein mRNA levels in the heart. Clin Exp Pharmacol Physiol 2011; 38: 623-31. 